\title{
Newtonian Heating Effects of Oldroyd-B Liquid Flow with Cross-Diffusion and Second Order Slip
}

\author{
K. Loganathan ${ }^{1(\bowtie)}$, K. Tamilvanan ${ }^{2}$, Amelec Viloria ${ }^{3,4}$, Noel Varela ${ }^{3}$, \\ and Omar Bonerge Pineda Lezama ${ }^{5}$ \\ 1 Department of Mathematics, Faculty of Engineering, \\ Karpagam Academy of Higher Education, Coimbatore 641021, Tamilnadu, India \\ loganathankaruppusamy304@gmail.com \\ 2 Department of Mathematics, Government Arts College for Men, \\ Krishnagiri 635001, Tamilnadu, India \\ 3 Universidad de la Costa, Barranquilla, Colombia \\ 4 Universidad Peruana de Ciencias Aplicadas, Lima, Peru \\ ${ }^{5}$ Universidad Tecnológica Centroamericana (UNITEC), San Pedro Sula, Honduras
}

\begin{abstract}
The current study highlights the Newtonian heating and second-order slip velocity with cross-diffusion effects on Oldroyd-B liquid flow. The modified Fourier heat flux is included in the energy equation system. The present problem is modeled with the physical governing system. The complexity of the governing system was reduced to a nonlinear ordinary system with the help of suitable transformations. A homotopy algorithm was used to validate the nonlinear system. This algorithm was solved via MATHEMATICA software. Their substantial aspects are further studied and reported in detail. We noticed that the influence of slip velocity order two is lower than the slip velocity order one.
\end{abstract}

Keywords: Oldroyd-B liquid $\cdot$ Second order slip $\cdot$ Cross diffusion effects $\cdot$ Convective heating $\cdot$ Cattaneo-Christov heat flux

\section{Introduction}

Heat transport through non-Newtonian fluids is the significant study in recent times because of its industrial and engineering applications. Oldroyd-B fluid is one of the types of non-Newtonian fluids. This fluid contains viscoelastic behaviour. Loganathan et al. [1] exposed the 2nd-order slip phenomena of Oldroyd-B fluid flow with cross diffusion impacts. Hayat et al. [2] performed the modified heat flux impacts with multiple chemical reactions on Oldroyd-B liquid flow. Eswaramoorthi et al. [3] studied the influence of cross-diffusion on viscoelastic liquid induced by an unsteady stretchy sheet. Elanchezhian et al. [4] examined the important facts of swimming motile microorganisms with stratification effects on Oldroyd-B fluid flow. Loganathan and Rajan [5] explored the entropy effects of Williamson nanoliquid caused by a stretchy plate with partial 
slip and convective surface conditions. The innovative research articles on nonNewtonian fluid flow with different geometry's and situations are studied in ref's [6-10].

As far as our survey report the Newtonian heating effects along with slip order two on Oldroyd-B liquid flow is not examined yet. The present study incorporates the cross diffusion and modified Fourier heat flux into the problem. The eminent homotopy technique [11-13] is employed for computing the ODE system and the results are reported via graphs.

\section{$2 \quad$ Modeling}

We have constructed the Oldroyd-B liquid flow subjected to below stated aspects:

1. Incompressible flow

2. Second-order velocity slip

3. Magnetic field

4. Binary chemical reaction

5. Stretching plate with linear velocity.

6. Cross-diffusion effects

7. Modified Fourier heat flux

Figure 1 represents graphical illustration of physical problem. The governing equations are stated below:

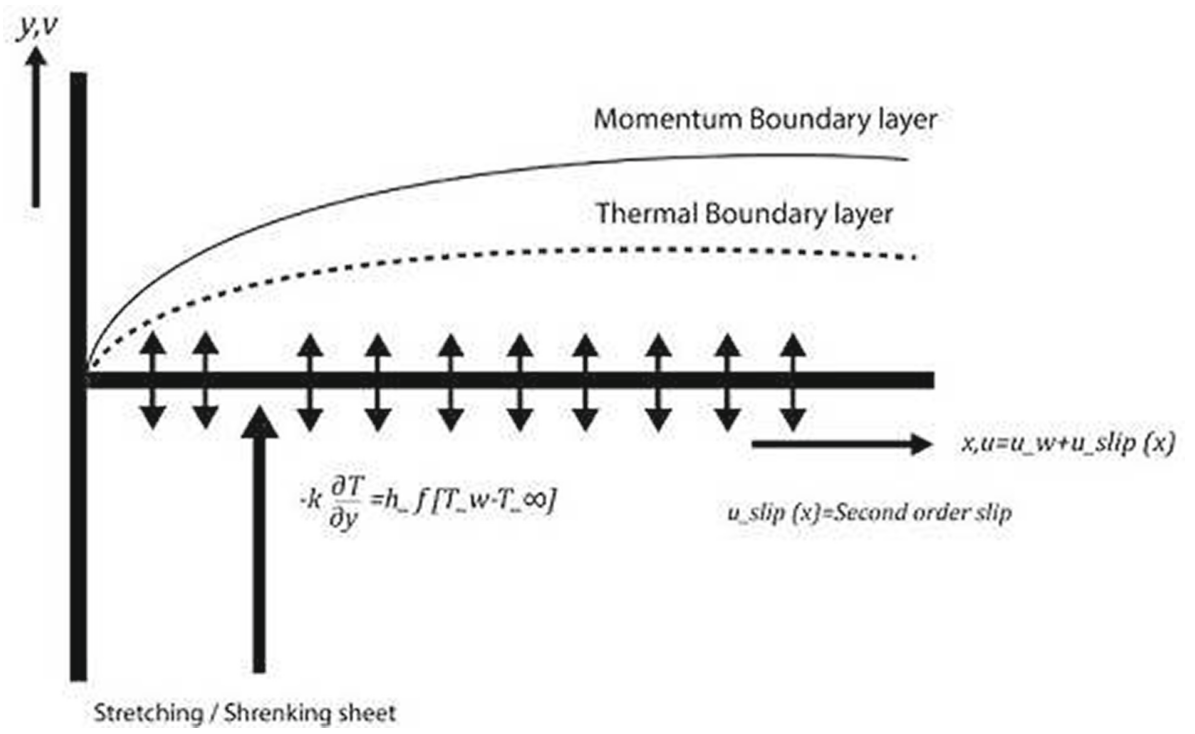

Fig. 1. Schematic diagram 


$$
\begin{aligned}
& \frac{\partial u}{\partial x}+\frac{\partial v}{\partial y}=0 \\
& \frac{\partial u}{\partial x} u+\frac{\partial u}{\partial y} v+A_{1}\left(u^{2} \frac{\partial^{2} u}{\partial x^{2}}+v^{2} \frac{\partial^{2} u}{\partial y^{2}}+2 u v \frac{\partial^{2} u}{\partial x \partial y}\right)=\mu \frac{\partial^{2} u}{\partial y^{2}} \\
& \quad-\mu A_{2} v \frac{\partial^{3} u}{\partial y^{3}}-\frac{\partial u}{\partial x} \frac{\partial^{2} u}{\partial y^{2}}-\frac{\partial u}{\partial y} \frac{\partial^{2} v}{\partial y^{2}}+\left(u \frac{\partial^{3} u}{\partial x \partial y^{2}}\right)-\frac{\sigma B_{0}^{2}}{\rho}\left(u+A_{1} v \frac{\partial u}{\partial y}\right) \\
& \frac{\partial T}{\partial x} u+\frac{\partial T}{\partial y} v=\frac{k}{\rho c_{p}} \frac{\partial^{2} T}{\partial y^{2}}-\frac{1}{\rho c_{p}} \frac{\partial q_{r}}{\partial y}+\frac{D_{m} k_{T}}{c_{s} c_{p}} \frac{\partial^{2} C}{\partial y^{2}} \\
& \frac{\partial C}{\partial x} u+\frac{\partial C}{\partial y} v=D_{m} \frac{\partial^{2} C}{\partial y^{2}}+\frac{D_{m} k_{T}}{T_{m}} \frac{\partial^{2} T}{\partial y^{2}}-k_{m}\left(C-C_{\infty}\right)
\end{aligned}
$$

The boundary points are

$$
\begin{aligned}
& u=u_{w}+u_{\text {slip }}=a x+\lambda_{1} \frac{\partial u}{\partial y}+\lambda_{2} \frac{\partial^{2} u}{\partial y^{2}}, \quad v=0, \\
& -k \frac{\partial T}{\partial y}=h_{f} T, \quad C=C_{w} \quad \text { at } y=0, \\
& u(\rightarrow 0), \quad v(\rightarrow 0), \quad T\left(\rightarrow T_{\infty}\right), \quad C\left(\rightarrow C_{\infty}\right) \text { as } y(\rightarrow \infty),
\end{aligned}
$$

where $A_{1}$ (= relaxation time), $A_{2}$ (= retardation time), $B_{0}$ (= constant magnetic field), $a$ (=stretching rate), $c_{p}$ (= specific heat), $c_{\infty}$ (= ambient concentration), $c_{w}$ (= fluid wall concentration), $D_{m}$ (= diffusion coefficient), $k$ (=thermal conductivity), $T_{\infty}$ (= ambient temperature), $T_{w}$ (= convective surface temperature), $u, v$ (=Velocity components), $u_{w}$ (= velocity of the sheet), $\lambda_{1}$ (=first order slip velocity factor), $\lambda_{2}$ (= second order slip velocity factor), $\mu$ (=kinematic viscosity), $\rho$ (=density), $\sigma$ (= electrical conductivity), $\gamma$ (=dimensionless thermal relaxation time). The energy equation updated with Cattaneo-Christov heat flux is defined as:

$$
\begin{aligned}
u \frac{\partial T}{\partial x}+v \frac{\partial T}{\partial y} & +2 u v \frac{\partial T^{2}}{\partial x \partial y}+\lambda\left(u^{2} \frac{\partial^{2} T}{\partial x^{2}}+v^{2} \frac{\partial^{2} T}{\partial y^{2}}+\left(u \frac{\partial u}{\partial x}+v \frac{\partial u}{\partial y}\right) \frac{\partial T}{\partial x}\right. \\
+ & \left.\left(u \frac{\partial v}{\partial x}+v \frac{\partial v}{\partial y}\right) \frac{\partial T}{\partial y}\right)=\frac{k}{\rho c_{p}} \frac{\partial^{2} T}{\partial y^{2}}-\frac{1}{\rho c_{p}} \frac{\partial q_{r}}{\partial y}+\frac{D_{m} k_{T}}{c_{s} c_{p}} \frac{\partial^{2} C}{\partial y^{2}}
\end{aligned}
$$

The transformations are

$$
\begin{aligned}
& \psi=\sqrt{a \mu} x f(\eta), u=\frac{\partial \psi}{\partial y}, v=-\frac{\partial \psi}{\partial x}, \eta=\sqrt{\frac{a}{\mu} y} \\
& v=-\sqrt{a \mu} f(\eta), \quad u=a x f^{\prime}(\eta), \quad \phi(\eta)=\frac{C-C_{\infty}}{C_{w}-C_{\infty}}, \theta(\eta)=\frac{T-T_{\infty}}{T_{w}-T_{\infty}},
\end{aligned}
$$


From the above transformations we derive the ODE system as follows,

$$
\begin{aligned}
& f^{\prime \prime \prime}+\beta\left(f^{\prime \prime 2}-f f^{i v}\right)+f f^{\prime \prime}-f^{\prime 2}+\alpha\left(2 f f^{\prime} f^{\prime \prime}-f^{2} f^{\prime \prime \prime}\right)-M\left(f^{\prime}-\alpha f f^{\prime \prime}\right)=0 \\
& f \theta^{\prime}-\gamma\left(f^{2} \theta^{\prime \prime}+f f^{\prime} \theta^{\prime}\right)+\frac{1}{\operatorname{Pr}}\left(1+\frac{4}{3} R d\right) \theta^{\prime \prime}+D_{f} \phi^{\prime \prime}=0 \\
& \frac{1}{S c} \phi^{\prime \prime}+f \phi^{\prime}-C r \phi+\operatorname{Sr} \theta^{\prime \prime}=0
\end{aligned}
$$

with boundary points

$$
\begin{aligned}
& f(0)=0, f^{\prime}(0)=1+\epsilon_{1} f^{\prime \prime}(0)+\epsilon_{2} f^{\prime \prime \prime}(0), \quad \theta^{\prime}(0)=-N w(1+\theta(0)), \quad \phi(0)=1 \\
& f^{\prime}(\infty)=0, \quad \theta(\infty)=0, \quad \phi(\infty)=0,
\end{aligned}
$$

The variables are defined as:

$\epsilon_{1}=($ first order velocity constant $)=\lambda_{1} \sqrt{a / \mu} ; \epsilon_{2}=$ (second order velocity constant $)=\lambda_{2} \frac{a}{\mu} \frac{h_{f}}{k} \sqrt{\mu / a} ; \alpha=($ relaxation time constant $)=A_{1} a ; \quad \beta=($ retardation time constant $)=A_{2} a ; \mathrm{M}=($ magnetic field constant $)=\frac{\sigma B_{0}^{2}}{\rho a} ; \operatorname{Pr}=($ Prandtl number $)=\frac{\rho C_{p}}{k} ; R d=($ radiation constant $)=\frac{4 \sigma^{*} T_{\infty}^{3}}{k k^{*}} ; \gamma=\lambda a ; D_{f}=($ Dufour number $)=\frac{D_{m} k_{T}}{\mu c_{s} c_{p}} \frac{c_{w}-c_{\infty}}{T_{w}-T_{\infty}} ; C r=($ chemical reaction constant $)=\frac{k_{m}}{a} ; S c=($ Schmidt number $)=\frac{\mu}{D_{m}} ; S r=($ Soret number $)=\frac{D_{m} k_{T}}{\mu T_{m}} \frac{T_{w}-T_{\infty}}{c_{w}-c_{\infty}}$.

\section{Solution Methodology}

We using the homotopy technique for validate the convergence of the nonlinear systems. The basic guesses and linear operators are defined as:

$$
\begin{aligned}
& f_{0}=\eta e^{-\eta}+\frac{3 \epsilon_{2}-2 \epsilon_{1}}{\epsilon_{2}-1-\epsilon_{1}} * e^{-\eta}-\frac{3 \epsilon_{2}-2 \epsilon_{1}}{\epsilon_{2}-1-\epsilon_{1}}, \quad \phi_{0}=e^{-\eta}, \quad \theta_{0}=\frac{N w * e^{-\eta}}{1-N w} \\
& L_{f}=f^{\prime}\left(f^{\prime \prime}-1\right), \quad L_{\phi}=\left(\phi^{\prime \prime}\right)-(\phi), L_{\theta}=\left(\theta^{\prime \prime}\right)-(\theta) .
\end{aligned}
$$

which satisfies the property

$L_{f}\left[D_{1}+D_{2} e^{\eta}+D_{3} e^{-\eta}\right]=0, \quad L_{\phi}\left[D_{6} e^{\eta}+D_{7} e^{-\eta}\right]=0, \quad L_{\theta}\left[D_{4} e^{\eta}+D_{5} e^{-\eta}\right]=0$,

where $D_{k}(k=1--7)$ are constants. The special solutions are

$$
\begin{aligned}
& f_{m}(\eta)=f_{m}^{*}(\eta)+D_{1}+D_{2} e^{\eta}+D_{3} e^{-\eta} \\
& \phi_{m}(\eta)=\phi_{m}^{*}(\eta)+D_{6} e^{\eta}+D_{7} e^{-\eta} \\
& \theta_{m}(\eta)=\theta_{m}^{*}(\eta)+D_{4} e^{\eta}+D_{5} e^{-\eta}
\end{aligned}
$$

In Fig. 2 the straight lines are named as h-curves. The permissible range of $h_{f}, h_{\theta} \& h_{\phi}$ are $-1.7 \leq h_{f} \leq-0.6,-1.2 \leq h_{\theta} \leq-0.2,-1.2 \leq h_{\phi} \leq-0.2$, respectively. Order of convergent series is depicted in Table 1 . Table 2 depicts $f^{\prime \prime}(0)$ in the special case $M=\beta=0$. It is noted that the $f^{\prime \prime}(0)$ values are well matched with the previous reports [14-16]. 


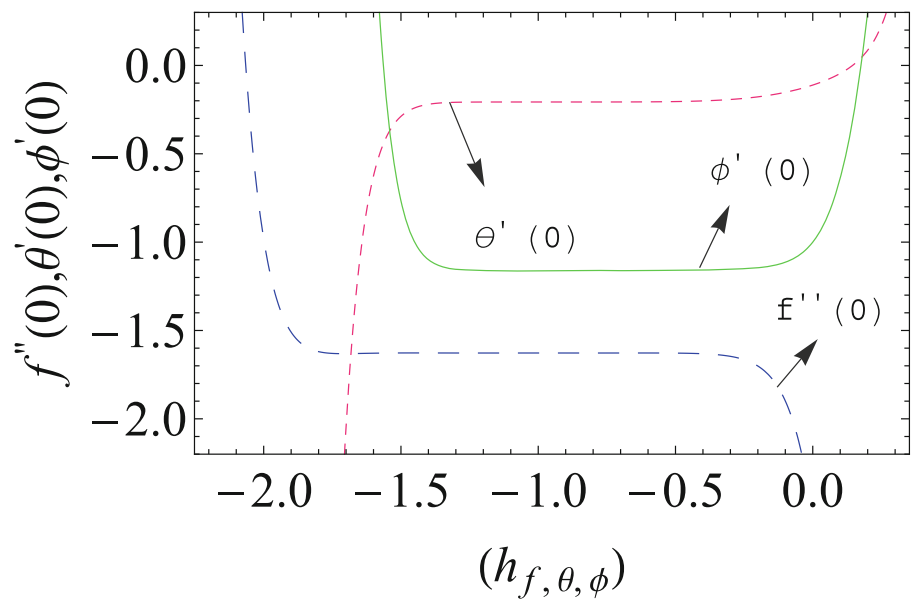

Fig. 2. $h$-curves for $h_{f}, h_{\theta}, h_{\phi}$

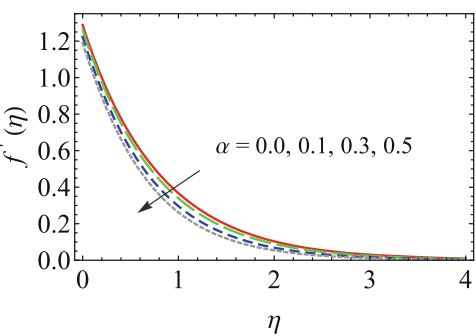

(a)

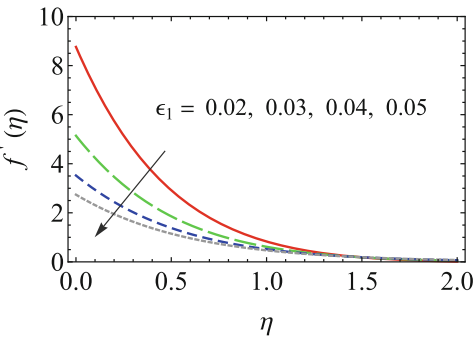

(c)

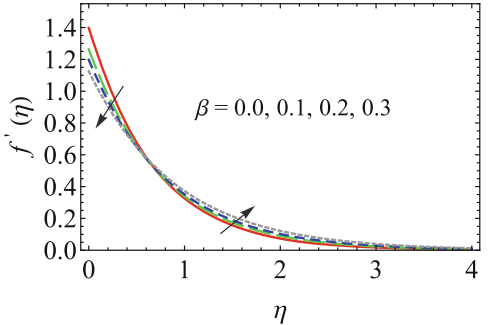

(b)

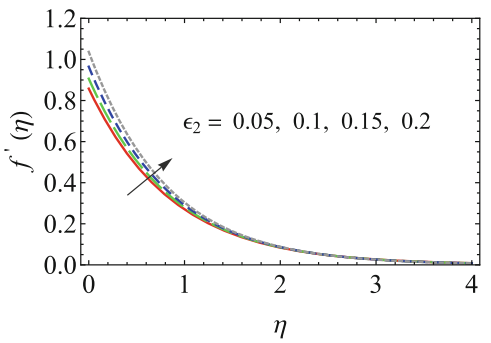

(d)

Fig. 3. $f^{\prime}(\eta)$ for various range of parameters $\left(\alpha, \beta, \epsilon_{1}, \epsilon_{2}\right)$.

\section{Results and Discussion}

Physical Characteristics of rising parameters versus, Concentration $\phi(\eta)$, velocity $f(\eta)$ and temperature $\theta(\eta)$ are investigated in Figs. 3, 4 and 5. Figure 3 depicted the velocity distribution $f(\eta)$ for different range of $\alpha, \beta, \epsilon_{1}, \epsilon_{2}$. It is noted that the velocity reduces for $\beta$ and $\epsilon_{1}$, while it increases for $\alpha$ and $\epsilon_{2}$. The temperature 


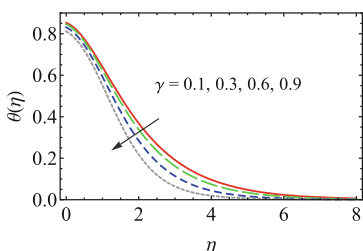

(a)

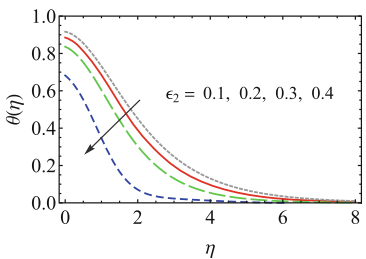

(d)

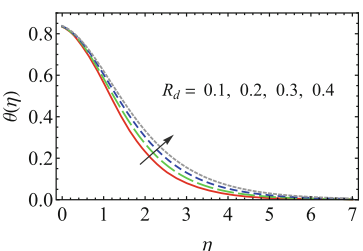

(b)

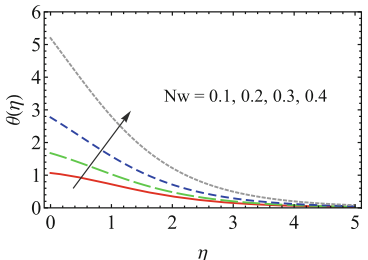

(e)

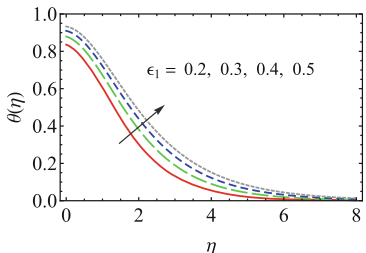

(c)

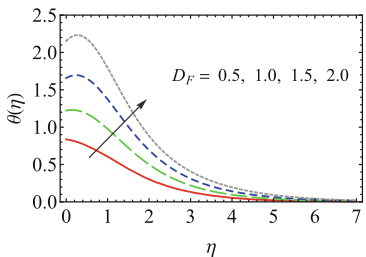

(f)

Fig. 4. $\theta(\eta)$ for various range of parameters $\left(\gamma, R_{d}, \epsilon_{1}, \epsilon_{2}, N w\right.$ and $\left.D_{F}\right)$.

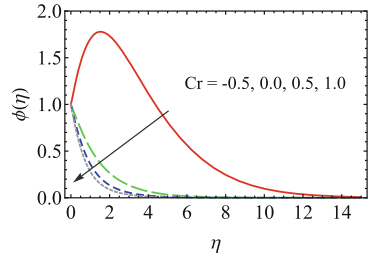

(a)

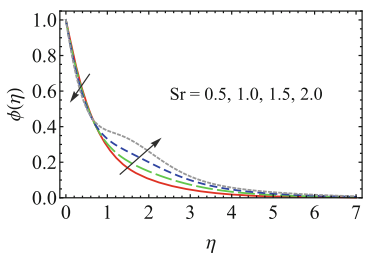

(b)

Fig. 5. $\phi(\eta)$ for various range of parameters $(\mathrm{Cr}$ and $\mathrm{Sr})$.

Table 1. Approximations for convergence

\begin{tabular}{c|l|l|l}
\hline Order & $-f^{\prime \prime}(0)$ & $-\theta^{\prime}(0)$ & $-\phi^{\prime}(0)$ \\
\hline 2 & 1.8126 & 0.1532 & 1.1289 \\
\hline 7 & 1.6331 & 0.1979 & 1.1558 \\
\hline 12 & 1.6273 & 0.2059 & 1.1606 \\
\hline 17 & 1.6274 & 0.2068 & 1.1616 \\
\hline 22 & 1.6274 & 0.2068 & 1.1616 \\
\hline 27 & 1.6274 & 0.2068 & 1.1616 \\
\hline 35 & 1.6274 & 0.2068 & 1.1616
\end{tabular}

distribution $\theta(\eta)$ for different range of $\gamma, R_{d}, \epsilon_{1}, \epsilon_{2}, N w$ and $D_{F}$ are sketched in Fig. 4. Thermal boundary layer decays with increasing the $\gamma$ and $\epsilon_{2}$ values. Larger values of $R_{d}, \epsilon_{1}$ and $D_{F}$ boosts the temperature distribution $\theta(\eta)$. Figure 5 
Table 2. Validation of $f^{\prime \prime}(0)$ in the specific case for various $\alpha$ when $\beta=M=0$

\begin{tabular}{l|l|l|l|l}
\hline$\alpha$ & Ref. [14] & Ref. [15] & Ref. [16] & Present \\
\hline 0.0 & 1.000 & 0.9999963 & 1.00000 & 1.00000 \\
\hline 0.2 & 1.0549 & 1.051949 & 1.05189 & 1.05189 \\
\hline 0.4 & 1.10084 & 1.101851 & 1.10190 & 1.10190 \\
\hline 0.6 & 1.0015016 & 1.150162 & 1.15014 & 1.15014 \\
\hline 0.8 & 1.19872 & 1.196693 & 1.19671 & 1.19671 \\
\hline
\end{tabular}

shows the influence on $\phi(\eta)$ for various values of $C r$ and $S r$. These parameters shows the opposite effect in $\phi(\eta)$.

\section{Conclusion}

The salient outcomes the flow problem is given below:

1. Retardation time parameter $(\beta)$ is inversely proportional to the relaxation time parameter $(\alpha)$ is in velocity profile.

2. Thermal boundary layer enhances due to increasing the $R_{d}, N w, D_{F}$ whereas it decays for higher $\epsilon_{1}$ and $\gamma$.

3. Higher Soret number values enhance the solutal boundary thickness.

\section{References}

1. Loganathan, K., Sivasankaran, S., Bhuvaneshwari, M., Rajan, S.: Second-order slip, cross- diffusion and chemical reaction effects on magneto-convection of Oldroyd-B liquid using Cattaneo-Christov heat flux with convective heating. J. Therm. Anal. Calorim. 136, 401-409 (2019). https://doi.org/10.1007/s10973-018-7912-5

2. Hayat, T., Imtiaz, M., Alsaedi, A., Almezal, S.: On Cattaneo-Christov heat flux in MHD flow of Oldroyd-B fluid with homogeneous-heterogeneous reactions. J. Magn. Mater. 401(1), 296-303 (2016)

3. Eswaramoorthi, S., Sivasankaran, S., Bhuvaneswari, M., Rajan, S.: Soret and Dufour effects on viscoelastic boundary layer flow over a stretchy surface with convective boundary condition with radiation and chemical reaction. Sci. Iran B. 23(6), 2575-2586 (2016)

4. Elanchezhian, E., Nirmalkumar, R., Balamurugan, M., Mohana, K., Prabu, K.M.: Amelec Viloria: heat and mass transmission of an Oldroyd-B nanofluid flow through a stratified medium with swimming of motile gyrotactic microorganisms and nanoparticles. J. Therm. Anal. Calorim. (2020). https://doi.org/10.1007/s10973020-09847-w

5. Loganathan, K., Rajan, S.: An entropy approach of Williamson nanofluid flow with Joule heating and zero nanoparticle mass flux. J. Therm. Anal. Calorim. (2020). https://doi.org/10.1007/s10973-020-09414-3 
6. Bhuvaneswari, M., Eswaramoorthi, S., Sivasankaran, S., Hussein, A.K.: Crossdiffusion effects on MHD mixed convection over a stretching surface in a porous medium with chemical reaction and convective condition. Eng. Trans. 67(1), 3-19 (2019)

7. Loganathan, K., Sivasankaran, S., Bhuvaneswari, M., Rajan, S.: Dufour and Soret effects on MHD convection of Oldroyd-B liquid over stretching surface with chemical reaction and radiation using Cattaneo-Christov heat flux. IOP: Mater. Sci. Eng. 390, 012077 (2018)

8. Bhuvaneswari, M., Eswaramoorthi, S., Sivasankaran, S., Rajan, S., Saleh Alshomrani, A.: Effects of viscous dissipation and convective heating on convection flow of a second-grade liquid over a stretching surface: an analytical and numerical study. Sci. Iran. B 26(3), 1350-1357 (2019)

9. Muhammad, T., Alamri, S.Z., Waqas, H., et al.: Bioconvection flow of magnetized Carreau nanofluid under the influence of slip over a wedge with motile microorganisms. J. Therm. Anal. Calorim. (2020). https://doi.org/10.1007/s10973-02009580-4

10. Abbasbandy, S., Hayat, T., Alsaedi, A., Rashidi, M.M.: Numerical and analytical solutions for Falkner-Skan flow of MHD Oldroyd-B fluid. Int. J. Numer. Methods Heat Fluid Flow 24, 390-401 (2014)

11. Liao, S., Tan, Y.A.: General approach to obtain series solutions of nonlinear differential. Stud. Appl. Math. 119(4), 297-354 (2007)

12. Liao, S.J.: An explicit, totally analytic approximation of Blasius viscous flow problems. Int. J. Non-Linear Mech. 34, 759-778 (1999)

13. Loganathan, K., Mohana, K., Mohanraj, M., Sakthivel, P., Rajan, S., Impact of 3rd-grade nanofluid flow across a convective surface in the presence of inclined Lorentz force: an approach to entropy optimization. J. Therm. Anal. Calorim. (2020). https://doi.org/10.1007/s10973-020-09751-3

14. Sadeghy, K., Hajibeygi, H., Taghavi, S.M.: Stagnation-point flow of upperconvected Maxwell fluids. Int. J. Non-linear Mech. 41, 1242 (2006)

15. Mukhopadhyay, S.: Heat transfer analysis of the unsteady flow of a Maxwell fluid over a stretching surface in the presence of a heat source/sink. Chin. Phys. Lett. 29, 054703 (2012)

16. Abbasi, F.M., Mustafa, M., Shehzad, S.A., Alhuthali, M.S., Hayat, T.: Analytical study of Cattaneo-Christov heat flux model for a boundary layer flow of Oldroyd-B fluid. Chin. Phys. B. 25(1), 014701 (2016) 